\title{
A distributed approach to precoder selection using factor graphs for wireless communication networks
}

\author{
Igor M Guerreiro ${ }^{1 *}$, Dennis Hui ${ }^{2}$ and Charles C Cavalcante ${ }^{1}$
}

\begin{abstract}
This paper addresses distributed parameter coordination methods for wireless communication systems. This proposes a method based on a message-passing algorithm, namely min-sum algorithm, on factor graphs for the application of precoder selection. Two particular examples of precoder selection are considered: transmit antenna selection and beam selection. Evaluations on the potential of such an approach in a wireless communication network are provided, and its performance and convergence properties are compared with those of a baseline selfish/greedy approach. Simulation results for the precoder selection examples are presented and discussed, which show that the graph-based technique generally obtains gain in sum rate over the greedy approach at the cost of a larger message size. Besides, the proposed method usually reaches the global optima in an efficient manner. Methods of improving the rate of convergence of the graph-based distributed coordination technique and reducing its associated message size are therefore important topics for wireless communication networks.
\end{abstract}

Keywords: Distributed optimization, Message-passing algorithm, Factor graphs, Precoder selection

\section{Introduction}

In a cellular network, there are many occasions in which each cell needs to set a parameter value, such as reference signal, transmit power, beam direction, or scheduled user, in such a way that the setting is preferably in a compatible way with the settings of the neighboring cells in order to achieve a certain notion of optimality, such as maximizing the average system or user throughput, of the entire network [1,2]. The choice made by one cell on a local parameter often affects the interference level experienced by its immediate neighbors and hence their respective choices made on their local parameters, which in turn would influence the choices made by their neighbors' neighbors. The simplest example is perhaps the classical frequency reuse problem [3] where the frequency band in which each cell transmits or receives is preferably different from those of its immediate neighboring cells to avoid mutual interference [4].

\footnotetext{
*Correspondence: igor@gtel.ufc.br

${ }^{1}$ Wireless Telecommunication Research Group (GTEL), Universidade Federal

do Ceará (UFC), Fortaleza 60455-760, Brazil

Full list of author information is available at the end of the article
}

In some cases, such as the frequency reuse problem, the parameter is of relatively static nature and can be optimally planned and set before the network deployment. However, in other cases, such as the transmit power control or antenna/beam selection problems, the parameter is more dynamic and requires coordination to be continually performed. Therefore, a systematic methodology for coordinating the choices of any parameters across the network is desired. Moreover, in order to facilitate flexible, dense deployment of small base stations in future cellular networks, there is also an increased interest in methods of performing the coordination of parameters among neighboring cells in an autonomous and distributed fashion without a central controller, as any unplanned addition (or removal) of base stations can substantially alter the system topology and thus the preferred settings.

Factor graph and the associated sum-product algorithm have been widely used in probabilistic modeling of the relationship among interdependent (random) variables or parameters. There are numerous successful applications [5] including, most notably, various fast-converging algorithms for decoding low-density parity check codes

\section{Springer}

(c) 2013 Guerreiro et al. : licensee Springer. This is an Open Access article distributed under the terms of the Creative Commons Attribution License (http://creativecommons.org/licenses/by/2.0), which permits unrestricted use, distribution, and reproduction in any medium, provided the original work is properly cited. 
and turbo codes, generalized Kalman filtering, fast Fourier transform, etc. One classic example motivated the most: the prisoner's dilemma [6]. In a few words, the two prisoners find an equilibrium point if both greedily betray. However, this is not a good solution because they can individually decrease their sentences if both of them stay silent, which is the best solution, and it is reached only if there is a sort of communication or centralization. In this context, the graph-based approach reaches the optimal solution by applying the message-passing algorithm. Similar (but different) applications of factor graphs have also been recently proposed for the problem of fast beam coordination among base stations in [7-10]. The basic idea in those works is to model the relationship between the local parameters to be coordinated among different communication nodes of a network and their respective performance metrics or costs using a factor graph [5]. In $[7,8]$, the belief propagation algorithm is adopted to solve the downlink transmit beamforming problem in a multicell multiple-input-multiple-output (MIMO) system considering a one-dimensional cellular model. Moreover, in $[9,10]$ some message-passing algorithms (including the sum-product algorithm) are deployed to coordinate parameters of downlink beamforming in a distributed manner in a multicell single-input-single-output system.

In this work, we propose a method founded on the min-sum algorithm on factor graphs for the application of precoder selection (i.e., transmit antenna selection [11] and beam selection) in a distributed manner. Based on factor graphs, a variant of the sum-product algorithm [5], namely the min-sum algorithm [12], can then be applied in order for all nodes, through iterative message passing with their respective neighbor nodes, to decide upon the best set of local parameters that can collectively maximize a global performance metric across the network. The algorithm allows each communication node to be indecisive of its own decision until sufficient information about how its decision would affect the overall network performance is accumulated. The performance of such a graphbased method along with other distributed methods, e.g., game-theoretic approach [13], for coordination of discrete parameters in a wireless communication network is evaluated.

\section{Problem description}

Consider a communication network with $N$ communication nodes. A communication node described here may represent any communication device in general in a wireless communication network, for example, a base-station (BS), an access node, or a user equipment (UE) in a cellular communication system. Let $p_{i}$, for $i=1,2, \ldots, N$, denote a discrete parameter (or a vector of two or more discrete parameters) of the $i$ th communication node, whose value is drawn from a finite set $\mathcal{P}_{i}$ of $\left|\mathcal{P}_{i}\right|$ possible parameter values for that node, where $\left|\mathcal{P}_{i}\right|$ denotes the cardinality of $\mathcal{P}_{i}$, and let

$$
\mathbf{p} \equiv\left[\begin{array}{llll}
p_{1} & p_{2} & \cdots & p_{N}
\end{array}\right]^{T}
$$

be a vector collecting all the parameters in the network, where

$$
p_{i} \in \mathcal{P}_{i}, i=1,2, \ldots, N .
$$

Figure 1 shows a hexagon layout with $N=7$ communication nodes where the set $\mathcal{P}_{i}=\{1,2,3\}$, for $i=1,2, \ldots, 7$. Examples of discrete parameters are a precoding matrix index (PMI), which is largely used in 3GPP long-term evolution (LTE) systems and beyond to select precoding matrices $[13,14]$ to maximize the network throughput, and an index to a frequency band in a frequency reuse planning to minimize the number of collisions in frequency bands, just to name a few.

Each node $i$ is associated with a list $\mathcal{N}_{i}$ of proper neighbor nodes (i.e., excluding node $i$ ) whose choices of parameter values can affect the local performance of node $i$. For convenience, also let

$$
\mathcal{A}_{i} \equiv \mathcal{N}_{i} \cup\{i\}
$$

denote the 'inclusive' neighbor list or just the neighbor list of node $i$. Let $\mathbf{p}_{\mathcal{A}_{i}}$ denote the vector of those parameters of nodes in $\mathcal{A}_{i}$, with its ordering of parameters determined by the sorted indices in $\mathcal{A}_{i}$. Associated with each node $i$ is a performance metric or cost, denoted by $M_{i}\left(\mathbf{p}_{\mathcal{A}_{i}}\right)$, which is a function of those parameters in the neighbor list $\mathcal{A}_{i}$ of node $i$. Each node $i$ is assumed to be capable of communicating with all nodes in $\mathcal{A}_{i}$.

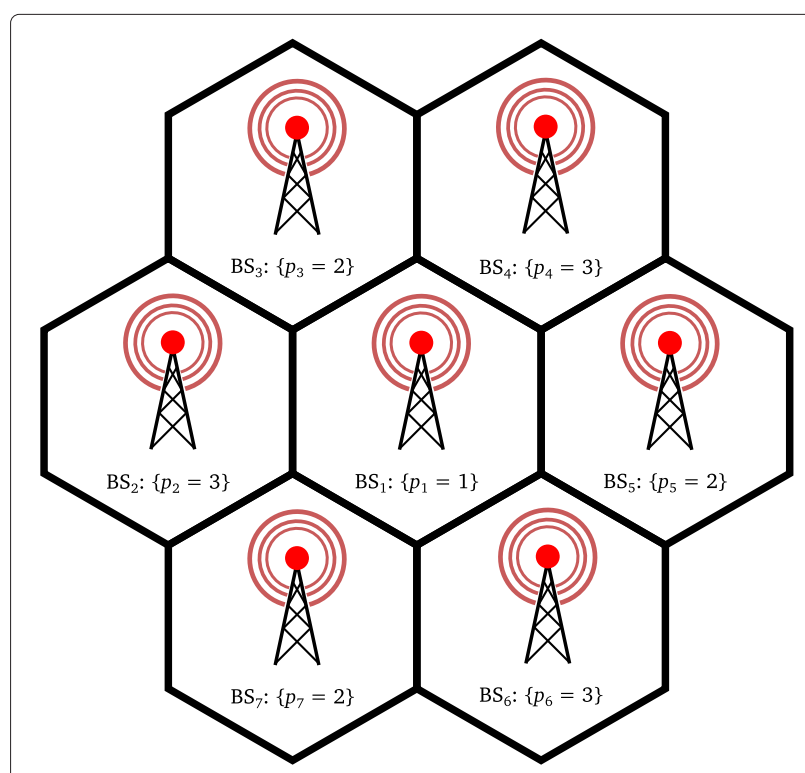

Figure $1 \mathrm{~A}$ general network example with a seven-cell hexagon layout. The parameter vector $\mathbf{p}=\left[\begin{array}{lllllll}1 & 3 & 2 & 3 & 2 & 3 & 2\end{array}\right]^{\top}$ is a possible status of the system, where $\mathcal{P}_{i}=\{1,2,3\}$ for $i=1,2, \ldots, 7$. 
Our goal is for each node $i$ to find, in a distributed fashion, its own optimal parameter $p_{i}^{*}$, which is the corresponding component of the optimal global parameter vector $\mathbf{p}^{*}$ that minimizes the total (global) performance metric given by

$$
M(\mathbf{p}) \equiv \sum_{i=1}^{N} M_{i}\left(\mathbf{p}_{\mathcal{A}_{i}}\right) .
$$

The problem of coordinating parameters can be solved adopting basically two types of solutions: (1) centralized approach, which yields the optimal global parameter vector, and (2) distributed approaches, which on one hand often provide suboptimal solutions through greedy techniques such as non-cooperative games, but on the other hand can provide near-optimal solutions by using a factor graph.

\section{Centralized solution}

Conceptually, the simplest approach to the optimization problem described above is to solve it jointly at a central location by direct computing

$$
\mathbf{p}_{C} \equiv\left[\begin{array}{c}
p_{C, 1} \\
p_{C, 2} \\
\cdots \\
p_{C, N}
\end{array}\right] \equiv \underset{\mathbf{p}}{\arg \min } \sum_{i=1}^{N} M_{i}\left(\mathbf{p}_{\mathcal{A}_{i}}\right),
$$

which is an optimal solution to the problem by definition. A major issue of this approach is its huge computational complexity for large network size $N$, as the complexity grows exponentially as the number of communication nodes increases, along with the inherent high signaling load (backhaul traffic) between the communication nodes and a central processing unit.

The computational complexity of the centralized solution is indeed very high. The minimum (or maximum) value of a cost (utility) function is usually found throughout all the combinations of the discrete parameters. The total number of combinations of parameters $c$ is given by

$$
c=\prod_{i=1}^{N}\left|\mathcal{P}_{i}\right| .
$$

For instance, if the network has $N=61$ nodes and $\left|\mathcal{P}_{i}\right|=$ 3 for $i=1,2, \ldots, N$, a number of $c \approx 10^{29}$ computations must be done to find the optimal value, which might be computationally prohibitive. Alternatively, the centralized technique may be replaced with the standard alternatingcoordinate optimization technique for dense networks. Such an approach starts with an arbitrary choice of $\mathbf{p}$ and iteratively optimizes each element (or a particular set of elements) of $\mathbf{p}$ one at a time while holding others fixed. Its convergence to the globally optimum result can be guaranteed under under some conditions, e.g., convex utility/cost function.

\section{Selfish/greedy solution}

Another approach to the optimization problem above is for each communication node to selfishly set its own parameter to optimize its own local performance based on the most recent choices made and given by its neighbors. In this approach, the complexity of each node grows only linearly with the cardinality of the set of parameters $\mathcal{P}_{i}$ since only the parameters of the node itself are considered in the optimization at the node. More precisely, in this approach, the local parameter $p_{i}$ at each communication node is iteratively chosen as

$$
\left.p_{S, i}^{(n+1)} \equiv \underset{p_{i}}{\arg \min } M_{i}\left(\mathbf{p}_{\mathcal{A}_{i}}\right)\right|_{\mathbf{p}_{\mathcal{N}_{i}}=\mathbf{p}_{S, \mathcal{N}_{i}}^{(n)}},
$$

where $n$ is the iteration index, $p_{S, i}^{(n+1)}$ denotes the choice of $p_{i}$ made at iteration $n+1$ using this selfish/greedy approach, and $\mathbf{p}_{S, \mathcal{N}_{i}}^{(n)}$ denotes the vector of those parameter choices made by nodes in $\mathcal{N}_{i}$ at the $n$th iteration. In turn, the parameter $p_{S, i}^{(n)}$ at each node is exchanged to its neighboring nodes so that every node obtains its parameter vector $\mathbf{p}_{S, \mathcal{N}_{i}}^{(n)}$ to compute its next parameter $p_{S, i}^{(n+1)}$.

From a game-theoretic perspective, the greedy solution may be seen as a non-cooperative game [6]. In this sense, the (greedy) game solution can be defined as a purestrategy Nash equilibrium (NE). In a few words, a NE is established if each player (i.e., each communication node) has chosen an action (i.e., a local parameter $p_{S, i}$ ) and no one can benefit from changing its action unilaterally while the others keep theirs unmodified. Therefore, an action tuple $\left\{p_{S, i}^{\star} \mathbf{p}_{S, \mathcal{N}_{i}}^{\star}\right\}$ for node $i$ is a NE if

$$
\begin{aligned}
& M_{i}\left(p_{S, i}^{\star}, \mathbf{p}_{S, \mathcal{N}_{i}}^{\star}\right) \geq M_{i}\left(p_{S, i}, \mathbf{p}_{S, \mathcal{N}_{i}}^{\star}\right), \\
& \forall p_{S, i} \in \mathcal{P}_{i}, i=1,2, \ldots, N .
\end{aligned}
$$

The superscript $\star$ denotes that the underlying parameter leads to a NE. The inequality above is a convenient form for representing a NE.

\section{Graph-based solution}

In the following, we describe another approach to the problem of minimizing the global metric in (1) by modeling the communication nodes and the associated local performance metrics using a factor graph. A factor graph is a bipartite graph consisting of a set of variable nodes and a set of factor nodes. Each variable node represents a variable and can only be connected to a factor node (but not another variable node) through an edge, while each factor node represents a factor which is a function of some of the variables. A factor node is connected to a variable node if and only if the corresponding function represented by the factor node depends on that variable.

Given a multivariate function, a factor graph expresses the mathematical structure of the factorization of such a 


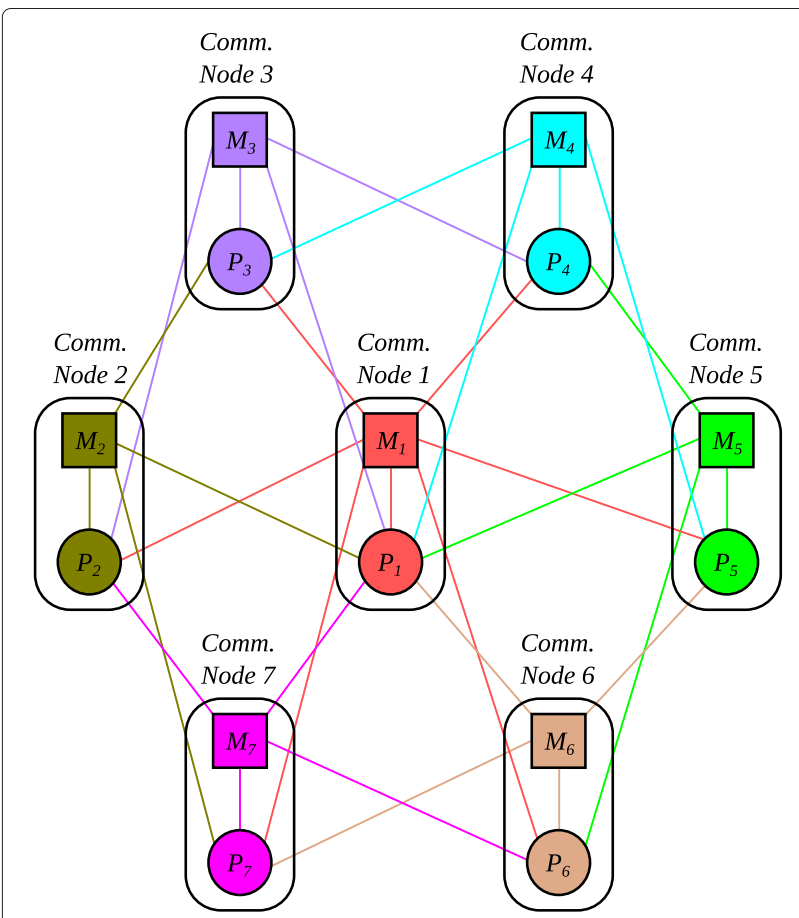

Figure 2 Factor graph model for a communication network with local parameters and local performance measures. own local parameter $p_{i}$ and those parameters $\left\{p_{i}\right\}_{i \in \mathcal{N}_{i}}$ of its neighbors upon which the metric $M_{i}\left(\mathbf{p}_{\mathcal{A}_{i}}\right)$ depends. For clarity, we use a different color to represent different communication nodes and the corresponding edges connecting their respective factor nodes with the relevant variable nodes.

The graph in Figure 2 can be also re-organized as in Figure 3, which clearly shows the bipartite property of the graph with factor nodes connected only to variable nodes through the respective edges. A message-passing algorithm, such as the sum-product algorithm, can then be executed on such a graph. Specific messages are computed and passed along each edge of the graph. Those messages that are passed on edges connecting factor and variable nodes of different colors correspond to information exchange between two neighboring communication nodes, while those messages that are passed on edges that connect nodes of the same color represent internal communications within each communication node. Each message depends only on the variable whose associated variable node is a vertex of the edge over which the message is passed along. More precisely, each message is simply a table of values with each entry corresponding to one of the possible values of the variable, as described in detail in the following.

\section{The graph-based distributed parameter coordination algorithm}

In this section, we describe the algorithm associated with the proposed distributed solution for the problem of parameter coordination. The sum-product algorithm [5] can be applied whenever the variables and functions associated with the factor graph are defined on a commutative semi-ring whose elements satisfy the distributive law. For our problem at hand, we apply the variant of sum-product algorithm that is based on the min-sum commutative semi-ring [12]. Recall that a semi-ring is a mathematical structure (e.g., a set) equivalent to a ring without an additive inverse. In a general way, the binary operations addition and multiplication can be replaced with others as long as the distributed law still holds. In this sense, a min-sum semi-ring simply replaces the addition operation with the minimum operation and the multiplication operation with the addition operation. In this case, edges to the respective variable nodes associated with its

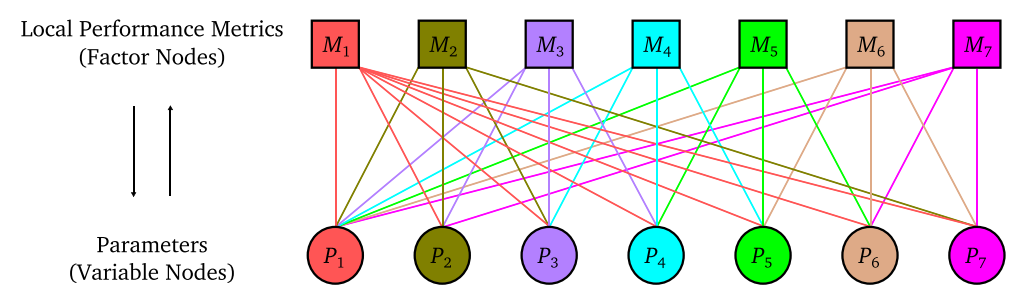

Figure 3 Re-organized factor graph for a communication network with local parameters and local performance measures. 
the sum-product algorithm is also called the min-sum algorithm.

More specifically, let $\mu_{M_{i} \rightarrow p_{k}}\left(p_{k}\right)$ denote the message to be passed from a factor node $v\left(M_{i}\right)$ to a variable node $v\left(p_{k}\right)$, and let $\mu_{p_{k} \rightarrow M_{i}}\left(p_{k}\right)$ denote the message to be passed from the variable node $v\left(p_{k}\right)$ to the factor node $v\left(M_{i}\right)$. Figure 4 shows the two kinds of messages passing on in a fragment of a factor graph. The min-sum algorithm, when applied to our problem at hand, simply iterates between the following two kinds of message computations and exchanges:

1. Factor node to variable node:

$$
\mu_{M_{i} \rightarrow p_{k}}\left(p_{k}\right)=\min _{\left.\mathbf{p} \mathcal{A}_{i} \backslash \backslash k\right\}}\left\{M_{i}\left(\mathbf{p}_{\mathcal{A}_{i}}\right)+\sum_{j \in \mathcal{A}_{i} \backslash\{k\}} \mu_{p_{j} \rightarrow M_{i}}\left(p_{j}\right)\right\},
$$

where the notation $\backslash\{k\}$ means that the underlying operator is performed over all associated variables except to variable $k$. To prevent messages from increasing endlessly, the messages are normalized to have zero mean.

2. Variable node to factor node:

$$
\mu_{p_{k} \rightarrow M_{i}}\left(p_{k}\right)=\sum_{j \in \mathcal{A}_{k} \backslash\{i\}} \mu_{M_{j} \rightarrow p_{k}}\left(p_{k}\right),
$$

which aggregates all the incoming messages at variable node $v\left(p_{k}\right)$ except to the one from factor node $v\left(M_{i}\right)$.

Here, an ideal error-free message pass is considered. The algorithm may begin with each factor node $v\left(M_{i}\right)$ computing outgoing message $\mu_{M_{i} \rightarrow p_{k}}\left(p_{k}\right)$ to $v\left(p_{k}\right)$ for each $k \in \mathcal{A}_{i}$ using (5) with all incoming messages $\mu_{p_{k} \rightarrow M_{i}}\left(p_{k}\right)$ from connected variable nodes initialized to unit messages, that is, messages with entries equal to zero for the problem at hand. It is worth mentioning that the initialization with unit messages may lead nodes to compute and propagate messages with equal entries. In

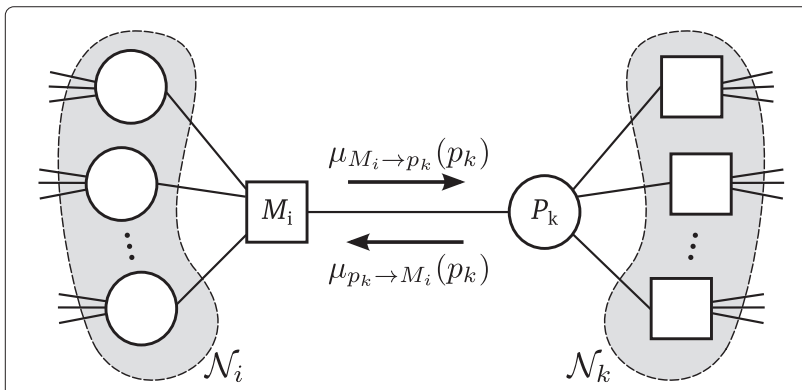

Figure 4 A factor-graph fragment, showing the message pass between factor node $M_{i}$ and variable node $p_{k}$. such situation, nodes are not capable of iteratively finding the best parameters as all the entries return the same cost. To circumvent this, the initial incoming messages $\mu_{p_{k} \rightarrow M_{i}}\left(p_{k}\right)$ can be initialized to random values close to zero.

Upon receipt of the message $\mu_{M_{i} \rightarrow p_{k}}\left(p_{k}\right)$, each variable node $v\left(p_{k}\right)$ then computes outgoing message $\mu_{p_{k} \rightarrow M_{i}}\left(p_{k}\right)$ to $v\left(M_{i}\right)$ for each $i \in \mathcal{A}_{k}$. Those new messages $\mu_{p_{k} \rightarrow M_{i}}\left(p_{k}\right)$ can be conveniently normalized to avoid messages increasing endlessly. The parameter for communication node $i$ is determined at its variable node $v\left(p_{i}\right)$ by

$$
p_{i}^{*}=\underset{p_{i}}{\arg \min }\left\{\sum_{j \in \mathcal{A}_{i}} \mu_{M_{j} \rightarrow p_{i}}\left(p_{i}\right)\right\} .
$$

The algorithm then iterates until a stopping criterion is reached, either a predetermined maximum number of iteration $\lambda$ or when the set of parameters computed in (7) converges to a fixed state, that is, the updated messages are equal to the previous computed messages, or equivalently,

$$
p_{i}^{(n+1)}=p_{i}^{(n)}, \quad \forall i=1,2, \ldots, N
$$

where $n$ is an iteration index such that $n \leq \lambda$.

Note that both messages computed in (5) and (6) depend only on the value of $p_{k}$. Since $p_{k} \in \mathcal{P}_{k}$ and $\mathcal{P}_{k}$ is assumed to be discrete and finite, each of the messages can be represented by a table of $\left|\mathcal{P}_{k}\right|$ entries.

In particular, the computation in (6) is just adding up the corresponding entries of multiple tables of the same size together. We summarize the proposed graph-based distributed coordination method from each communication node's perspective in Table 1 . Note that to reduce computational complexity, the minimization operation in (5) in step 2 can be computed through the standard alternating-coordinate optimization technique, although its convergence may not be guaranteed in this case.

When the factor graph contains no cycle (i.e., a closed path in the graph), it can be shown [5] that the messagepassing algorithm described above will yield the exact optimal solution that optimizes (1) in a single iteration. However, when it contains cycles, the algorithm has no natural termination and the messages pass multiple times on the edges of the factor graph in an iterative manner. In this case, the algorithm typically yields good approximations to the true optimal solution [5].

Regarding convergence issues, an undesired effect, i.e., ping-pong effect, may be experienced by nodes during the iterative process. The ping-pong effect refers to the alternation between two (or more) possible values of a variable ad infinitum. To avoid the continuity of this state, some perturbation may be inserted in the system, such as the addition of noise or forcing an unexpected value of some variables. In this context, the randomness of a 
Table 1 Graph-based distributed parameter coordination algorithm

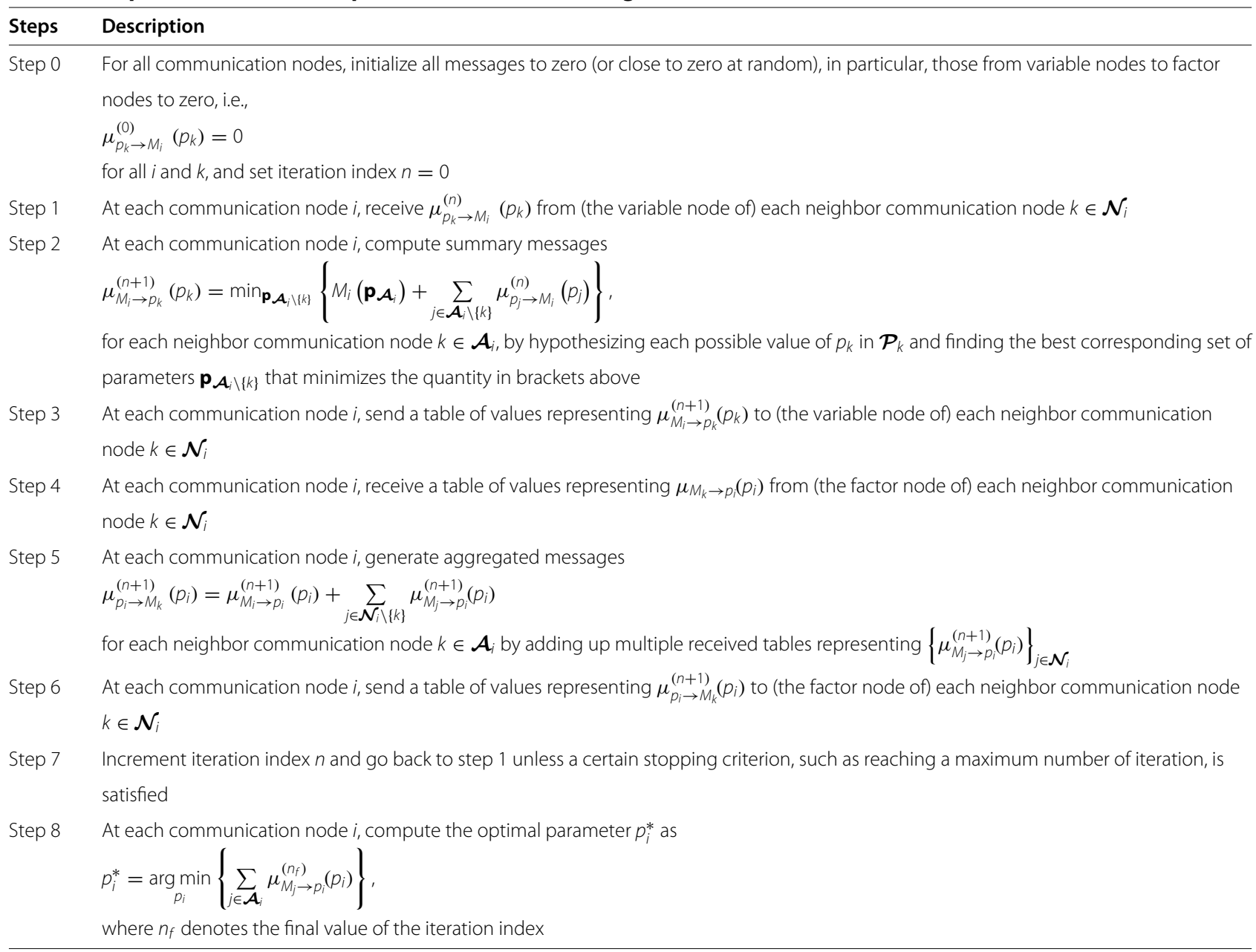

random scheduling, which is discussed below, acts as a perturbation in the system.

\section{Message-passing scheduling}

Regarding cases with cyclic graphs, the iterative process described above must follow a message-passing schedule to compute approximations of the true marginal functions. That is, the message-passing schedule must describe the way nodes are activated and the way messages are passed at each iteration. Note that the graphbased algorithm follows the message propagation rules previously described. In this work, two sorts of node scheduling based on the so-called flooding schedule [15] were considered: (1) random scheduling (RS), where each node has a certain probability of being activated to participate in the message pass at each iteration, and (2) simultaneous scheduling (SS), where all the nodes participate in the message pass at the same time at each iteration:

1. Random scheduling: In this scheduling mode, each node has two possibilities (which is similar to a 'coin flipping') at each iteration of the message-passing process: being either active or inactive. Let $P_{\text {active }}$ be the probability of being active, i.e., the chance of passing/receiving messages, during the iterative message-passing process. Thus, every node has $P_{\text {active }}$ $\%$ chance of being active at each iteration. If a node is inactive in a particular iteration, that node neither receives nor passes messages on its associated edges. That is, the messages associated with that node are not updated.

2. Simultaneous scheduling: In this scheduling mode, all the nodes pass/receive messages to/from their neighboring nodes, which is equivalent to set $P_{\text {active }}=1$ in the mode RS, i.e., $100 \%$ of the nodes active during the whole message-passing process.

Intuitively, the mode RS decreases the traffic of information among nodes because not all nodes are active during the message pass. In case of occurrence of the ping-pong effect, the inactivation of some nodes may perturb the system so that such an undesired effect ceases. 
On the other hand, the mode SS can reach a potential convergence point in a faster manner since all the nodes exchange messages. Thus, the choice of the messagepassing scheduling mode might depend on the network demand, such as latency and backhaul traffic. Moreover, the parameter $P_{\text {active }}$ may be optimized in order to either decrease the signaling load or speed up the convergence.

In the following, the application in which the proposed algorithm is evaluated as well as its associated parameters and performance metrics is presented.

\section{Precoder selection problem}

In this section, we discuss the parameter coordination problem, i.e., precoder selection, modeled by (1) in the context of wireless communications. Each parameter $p_{i}$ may represent a PMI for BS $i$ in the downlink (or UE $i$ in the uplink) in a cellular network, indicating which precoder from a predetermined set $\mathcal{P}_{i}$ of precoders or beamforming weights that $\mathrm{BS}$ (or UE) $i$ should use at a certain radio resource block to transmit signals. In practical systems, different UEs may be scheduled, and thus, different precoders may be used at different radio resource blocks. In this case, the coordination of precoders may be performed independently for each individual radio resource block.

Consider a multicell MIMO system in the downlink in which each BS has $N_{t}$ available transmit antennas and each item of UE has $N_{r}$ receive antennas. For convenience, each item of UE is simply referred to as a UE. The BS $i$ transmits precoded and spatially multiplexed vector $\mathbf{x}_{i}$ to its associated UE $i$. The vector $\mathbf{x}_{i}$ is defined as

$$
\mathbf{x}_{i}=\sqrt{\frac{1}{N_{s}}} \mathbf{W}\left(p_{i}\right) \mathbf{s}_{i}
$$

where $N_{s}$ is the number of data streams, $\mathbf{s}_{i}$ is the $N_{s} \times 1$ spatially multiplexed symbol vector, and $\mathbf{W}\left(p_{i}\right) \in \mathcal{W}$ is the $N_{t} \times N_{s}$ precoding matrix specified by the parameter $p_{i}$. Examples of set $\mathcal{W}$ can be a LTE precoder codebook, a fixed beamforming weight codebook, and a transmit antenna selection (TAS) codebook. In general, $\mathcal{W}$ is the set of all precoding matrices available for every communication node in the network. In order to index the elements of $\mathcal{W}$, assume an index set $\mathcal{I}$, which is equivalent to $\mathcal{P}_{i}$, defined as

$$
\mathcal{P}_{i} \equiv \mathcal{I} \triangleq\left\{1,2, \ldots,\left(\begin{array}{l}
N_{t} \\
N_{s}
\end{array}\right)\right\}
$$

for all the communication nodes. Then, a bijective function

$$
f: \mathcal{P}_{i} \leftrightarrow \mathcal{W}
$$

maps the elements of $\mathcal{P}_{i}$ onto the elements of $\mathcal{W}$ properly.
This work focuses on two particular examples of precoding matrix W: (1) TAS precoders and (2) discrete Fourier transform (DFT)-matrix-based precoders:

1. Transmit antenna selection: Let each element of $\mathcal{W}$ be an $N_{t} \times N_{s}$ submatrix of an identity matrix $\mathbf{I}_{N_{t}}$. That is, the unique non-null entry of each column of this submatrix selects a transmit antenna. For example, for $N_{t}=3$ and $N_{s}=2$,

$$
\mathcal{W}=\left\{\left[\begin{array}{ll}
1 & 0 \\
0 & 1 \\
0 & 0
\end{array}\right],\left[\begin{array}{ll}
1 & 0 \\
0 & 0 \\
0 & 1
\end{array}\right],\left[\begin{array}{ll}
0 & 0 \\
1 & 0 \\
0 & 1
\end{array}\right]\right\}
$$

where each matrix in $\mathcal{W}$ is a particular $\mathbf{W}$ indexed by $p_{i} \in \mathcal{P}_{i}$, according to (9) and (10). In other words, each matrix $\mathbf{W}$ selects two antennas out of three, where the first matrix selects antennas 1 and 2, the second matrix selects antennas 1 and 3 , and finally the last matrix selects antennas 2 and 3 .

2. Fixed-beam selection: Let each element of $\mathcal{W}$ be an $N_{t} \times N_{s}$ submatrix of the $N_{t} \times N_{t}$ DFT matrix $\mathbf{F}$ defined as being

$$
\mathbf{F}=\frac{1}{\sqrt{N_{t}}}\left[\begin{array}{ccccc}
1 & 1 & 1 & \cdots & 1 \\
1 & w & w^{2} & \cdots & w^{\left(N_{t}-1\right)} \\
1 & w^{2} & w^{4} & \cdots & w^{2\left(N_{t}-1\right)} \\
\vdots & \vdots & \vdots & \ddots & \vdots \\
1 & w^{\left(N_{t}-1\right)} & w^{2\left(N_{t}-1\right)} & \cdots & w^{\left(N_{t}-1\right)\left(N_{t}-1\right)}
\end{array}\right],
$$

where $w=e^{-\frac{2 \pi j}{N_{t}}}, e$ is the basis of the natural logarithm and $j$ is the imaginary unit. Basically, each matrix $\mathbf{W}$ changes the relative phase and direction of the vector $\mathbf{s}$ to be transmitted. For instance, for $N_{t}=3$ and $N_{s}=2$,

$$
\mathcal{W}=\frac{1}{\sqrt{3}}\left\{\left[\begin{array}{lc}
1 & 1 \\
1 & e^{-\frac{2 \pi j}{3}} \\
1 & e^{-\frac{4 \pi j}{3}}
\end{array}\right],\left[\begin{array}{lc}
1 & 1 \\
1 & e^{-\frac{4 \pi j}{3}} \\
1 & e^{-\frac{8 \pi j}{3}}
\end{array}\right],\left[\begin{array}{cc}
1 & 1 \\
e^{-\frac{2 \pi j}{3}} & e^{-\frac{4 \pi j}{3}} \\
e^{-\frac{4 \pi j}{3}} & e^{-\frac{8 \pi j}{3}}
\end{array}\right]\right\},
$$

where each matrix in $\mathcal{W}$, similar to the TAS case, is a particular $\mathbf{W}$ indexed by $p_{i} \in \mathcal{P}_{i}$, according to (9) and (10). In this example, there are three different beams to be selected, each one with a different combination of phase and direction.

The sampled incoming signal vector at the receiver $i$ is given as being

$$
\mathbf{y}_{i}=\sqrt{g_{i i}} \mathbf{H}_{i i} \mathbf{x}_{i}+\sum_{j \in \mathcal{N}_{i}} \sqrt{g_{j i}} \mathbf{H}_{j i} \mathbf{x}_{j}+\mathbf{v}_{i},
$$

where $\mathbf{H}_{j i}$ denotes the MIMO channel response from BS $j$ to the UE served by BS $i$ in the downlink, quasi-static over a data block, and $\mathbf{v}_{i}$ is a zero-mean circularly symmetric complex Gaussian noise vector. The constant $g_{j i}$ is 


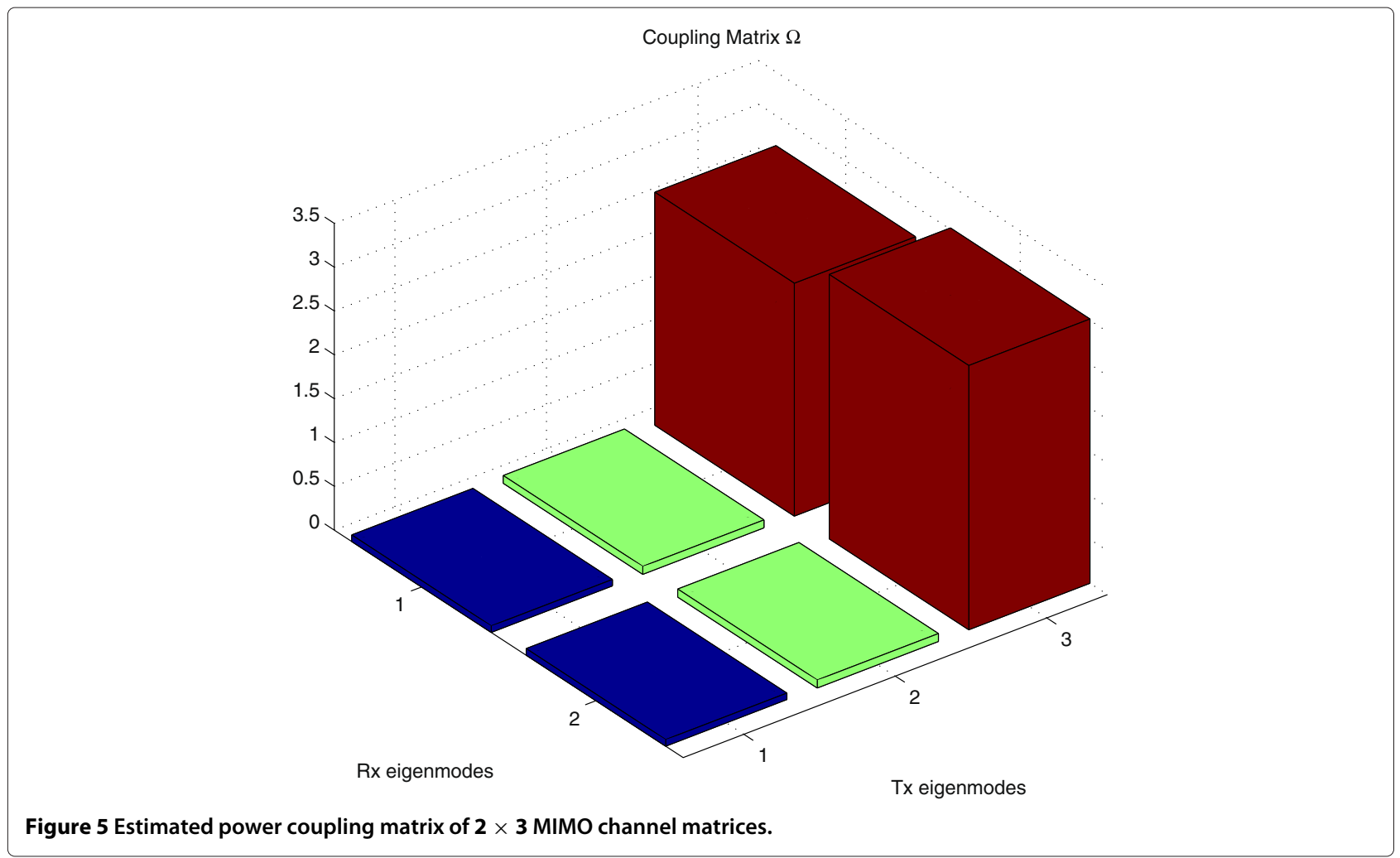

a gain that corresponds to the path loss of each signal, here modeled in a simplified way as being

$$
g_{j i}=\left(\frac{1}{d_{j i}}\right)^{\alpha}
$$

where the constant $\alpha$ refers to the path loss exponent and $d_{j i}$ is the distance between the transmitter $j$ and the receiver $i$. The second term on the right-hand side refers to the interference caused by the neighboring communication nodes. For each transmitter, the average transmit power is constant and given by

$$
\mathbb{E}\left\{\left\|\mathbf{x}_{i}\right\|^{2}\right\}=\frac{1}{N_{s}} \operatorname{tr}\left(\mathbf{W} \mathbf{W}^{H}\right)=P_{T},
$$

where $P_{T}$ is the average transmitted power in units of energy per signaling period. Also, the symbols are assumed to be uncorrelated, which means that $\mathbb{E}\left\{\mathbf{s}_{i} \mathbf{s}_{i}^{H}\right\}=$ $\mathbf{I}_{N_{s} \text {. }}$

In both cases, the local performance metric $M_{i}\left(\mathbf{p}_{\mathcal{A}_{i}}\right)$ may represent the negative of the data throughput $[16,17]$ of the cell corresponding to BS $i$ measured by

$$
M_{i}\left(\mathbf{p}_{\mathcal{A}_{i}}\right)=-\log \operatorname{det}\left(\mathbf{I}+\left|g_{i i}\right| \mathbf{R}_{i}^{-1} \mathbf{H}_{i i} \mathbf{W}\left(p_{i}\right) \mathbf{W}\left(p_{i}\right)^{H} \mathbf{H}_{i i}^{H}\right),
$$

where $\mathbf{R}_{i}$, defined herein as

$$
\mathbf{R}_{i} \triangleq \mathbf{R}_{\mathbf{v}_{i}}+\sum_{j \in \mathcal{N}_{i}}\left|g_{j i}\right| \mathbf{H}_{j i} \mathbf{W}\left(p_{j}\right) \mathbf{W}\left(p_{j}\right)^{H} \mathbf{H}_{j i}^{H},
$$

denotes the covariance matrix of the noise plus interference experienced by the UE served by BS $i$ in the downlink given that $\mathbf{R}_{\mathbf{v}_{i}}$ is the covariance matrix of the noise vector $\mathbf{v}_{i}$.

The global performance metric is simply the total data throughput in the network. Hence, the goal here is to

\section{Table 2 Parameters of simulation}

\begin{tabular}{lc}
\hline Parameters & Description/value \\
\hline Cell layout & Hexagon \\
Number of nodes & 7 or 61 \\
Number of PMls & 3 \\
Codebook & $(3 \times 2)$ TAS or DFT-matrix-based \\
Channel model & Measured-data-based channel \\
Spatial correlation & Yes \\
Pathloss exponent & 2.00 \\
Signal-to-noise ratio (dB) & 20 \\
Shadowing & No \\
UE positioning & Random \\
Intercell distance (m) & 500 \\
Parameter initialization & Random \\
Maximum number of iterations & 100 \\
per run & \\
Number of simulation runs & 85 or 1,000 \\
Message-passing scheduling & Simultaneous or random \\
type &
\end{tabular}




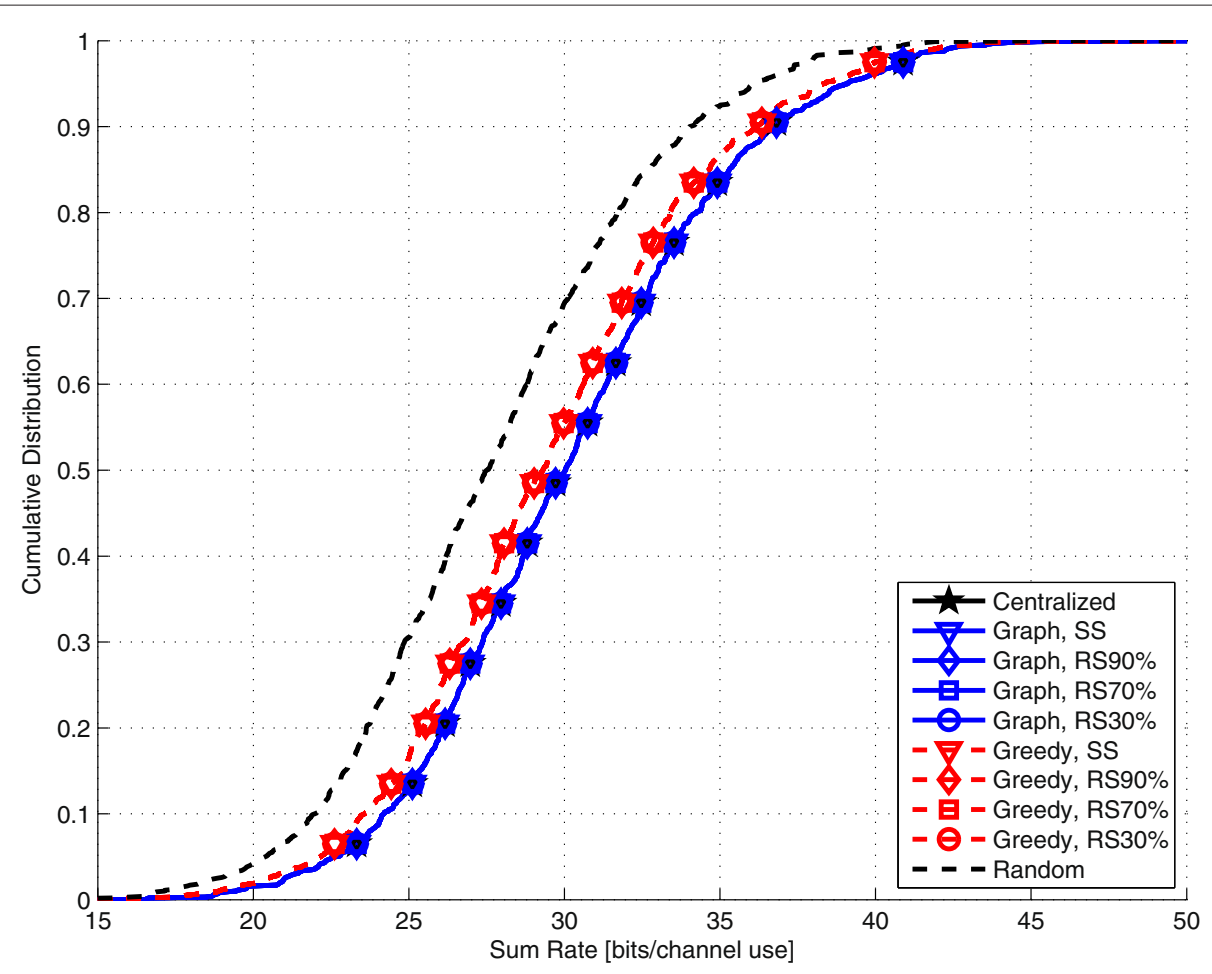

Figure 6 Performance analysis of graph-based technique for TAS problem in terms of sum rate in seven-node network.

employ a distributed algorithm for the BS to negotiate their choices of downlink precoding matrices with their respective neighbors so that the total data throughput in the network is maximized.

The channel response includes both a fast fading component and a path loss component, the latter determined by the distance between the corresponding BS and UE, according to (14). A basic system model is therefore needed to compute the relative distances between BS and UEs for each random drop of UEs in the cell grid considering fixed BSs' positioning. For convenience, the log-normal shadowing has not been modeled in this work.

To obtain more realistic results, each MIMO channel response was drawn from a data set $\mathcal{D}$ of measured channel matrices acquired by Ericsson Research during measurement campaigns made in Kista neighborhood in Stockholm, Sweden. The measurement campaigns were performed using a single BS placed on the roof of a building and a UE mounted inside a van at a convenient driving speed (see more details in $[18,19]$ ). A total of 324,000 samples of $2 \times 3$ channel matrices measured along a particular route of Kista compound the set $\mathcal{D}$. For the sake of removing any 'original' large-scale fading effect, each entry of the channel matrices was previously transformed into a zero-mean and unity-variance variable, such that

$$
\left[\mathbf{H}_{j i}\right]_{k, l}=\frac{\left[\mathbf{D}_{j i}\right]_{k, l}-\mu_{D}}{\sigma_{D}}, \quad \mathbf{D}_{j i} \in \mathcal{D}, k=1 \ldots 2, l=1 \ldots 3,
$$

where $\mathbf{D}_{j i}$, randomly picked up from set $\mathcal{D}$, is associated with receiver $j$ and transmitter $i, \mu_{D}$ and $\sigma_{D}^{2}$ are the mean and the variance of the entries of the matrices in $\mathcal{D}$, respectively, and $\mathbf{H}_{j i}$ is the transformed MIMO channel matrix also associated with receiver $j$ and transmitter $i$. The indices $k$ and $l$ index the element $(k, l)$ of both matrices $\mathbf{D}_{j i}$ and $\mathbf{H}_{j i}$. Then, the path loss modeled by the parameters $g_{j i}$ is in turn inserted to the matrix $\mathbf{H}_{j i}$ according to (13). It is worth noting that each element of $\mathcal{D}$ is randomly chosen only once so that each pair of receiver and transmitter has a different channel matrix. Particularly, the resulting channel matrices are characterized by the presence of a number of eigenmodes less than 2. Such a feature is observed in the estimated power

Table 3 Percent gain in sum rate for the TAS problem in a seven-node network

\begin{tabular}{|c|c|c|c|c|}
\hline \multirow{2}{*}{$\%$ Gain in sum rate over greedy } & \multirow[b]{2}{*}{ Simultaneous } & \multicolumn{3}{|c|}{ Message-passing scheduling } \\
\hline & & 90\% Random & $60 \%$ Random & $30 \%$ Random \\
\hline 50th CDF percentile & 2.465 & 2.465 & 2.465 & 2.465 \\
\hline
\end{tabular}




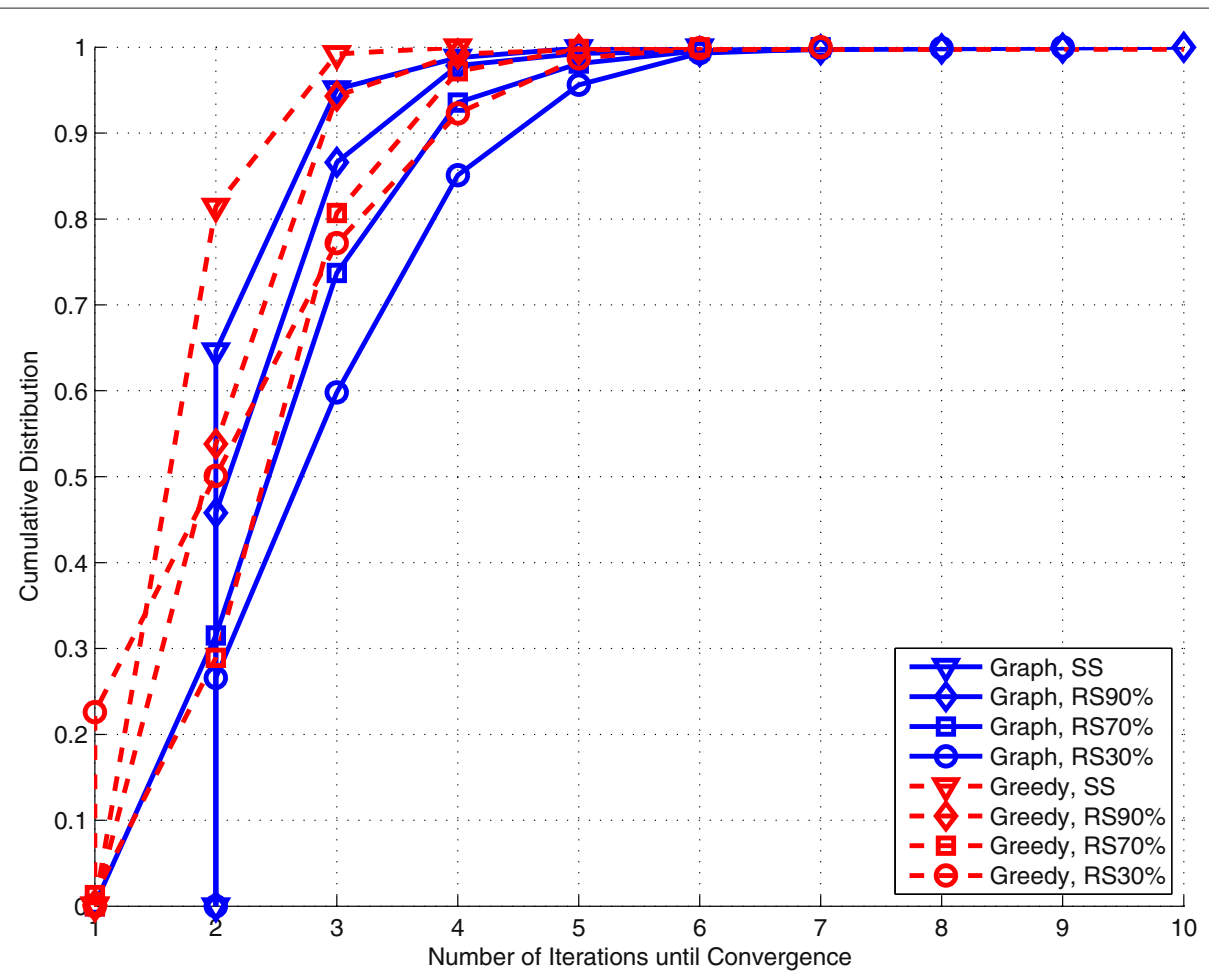

Figure 7 Convergence speed of graph-based technique against greedy technique for TAS problem in seven-node network.

coupling matrix $\Omega$ [20] of resulting MIMO channel matrices, which is shown in Figure 5. The matrix $\Omega$ shows the spatial arrangement of scattering objects between the transmitter and the receiver, where its columns refer to the transmit eigenmodes and the rows the receive eigenmodes. This matrix characterizes the entire data set $\mathcal{D}$. Consequently, as all the pairs of receiver and transmitter draw their channel matrices from $\mathcal{D}$, they observe the same spatial correlation. The motivation to use such a channel model is to provide a suitable scenario for the beam selection technique, which usually benefits from the characteristics of spatially correlated channel matrices.

Next, simulation results of the application of the proposed method in the precoder selection problems are shown and discussed.

\section{Simulation results}

In this section, the global performance metric presented in (16) in the precoder selection problem is investigated in order to evaluate how it behaves statistically in terms of cumulative distribution curves CDFs. Both examples of precoding selection, TAS and beam selection, are compared with the centralized solution, which is optimal by definition; the greedy solution, which is expected to provide a suboptimal result; and a lower bound provided by the random choice of the precoding matrix, kept constant during the simulation run, at each node. Moreover, the convergence speed, which is inversely proportional to the average number of iterations until convergence per simulation run, of both distributed approaches is qualitatively assessed in terms of CDF curves for only the cases where the underlying algorithm converges, following (4) and (8). Additionally, the convergence rate, defined as the ratio of the number of runs where the underlying algorithm converges to the total number of simulation runs, is shown for both distributed techniques. Both distributed algorithms are evaluated considering the different types of message-passing scheduling.

In order to evaluate the scalability of the proposed algorithm, a hexagon layout with $N=7$ or $N=61$ cells and a

Table 4 Convergence rate for the TAS problem in a seven-node network

\begin{tabular}{lcccc}
\hline \multirow{2}{*}{ Technique } & SS & \multicolumn{3}{c}{ Message-passing scheduling } \\
\cline { 2 - 5 } & 0.980 & RS, $\boldsymbol{P}_{\text {active }}=\mathbf{0 . 9}$ & RS, $\boldsymbol{P}_{\text {active }}=\mathbf{0 . 7}$ & RS, $\boldsymbol{P}_{\text {active }}=\mathbf{0 . 3}$ \\
\hline Graph-based & 0.985 & 0.993 & 1 \\
Greedy & 1 & 1 & 1 \\
\hline
\end{tabular}




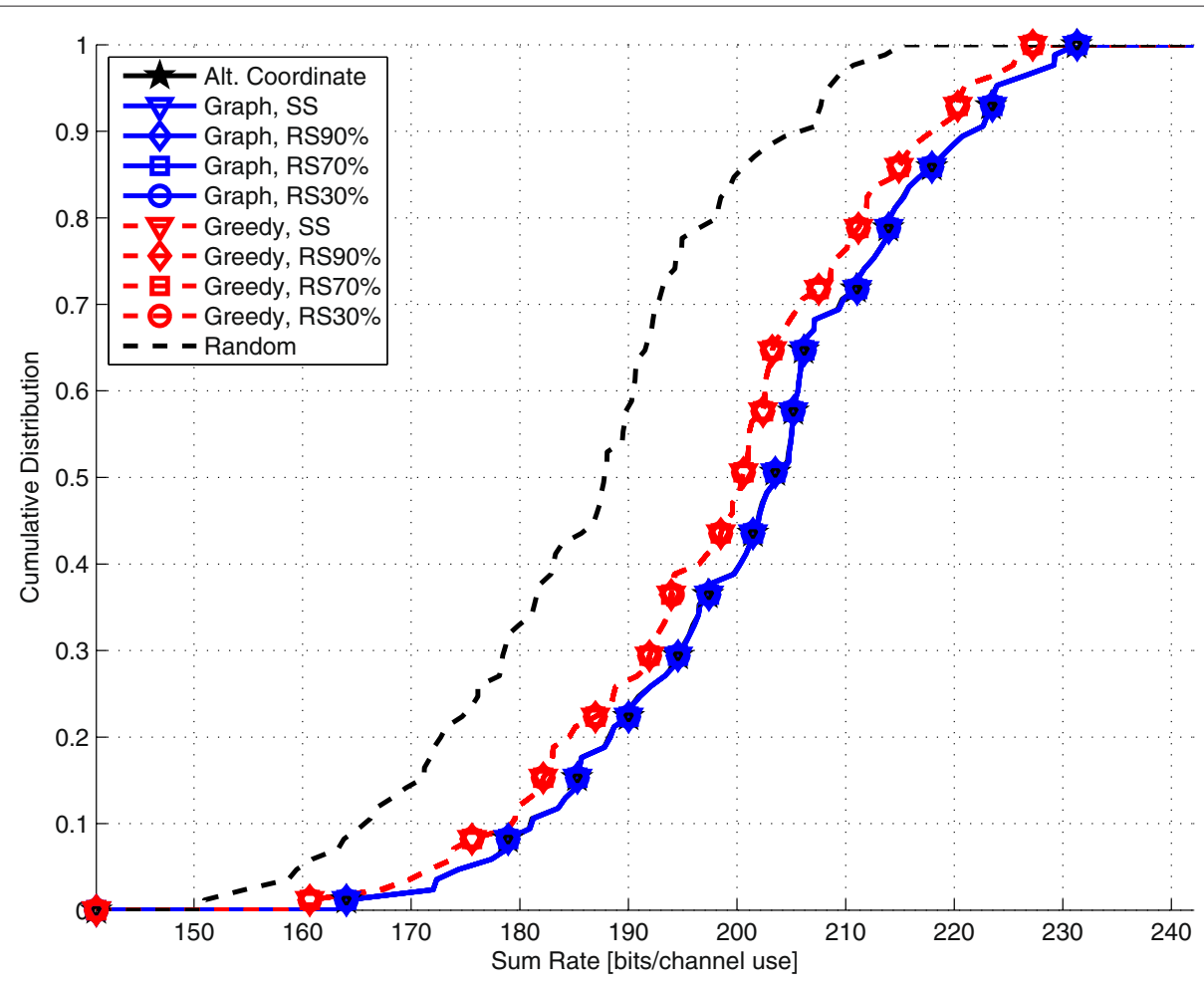

Figure 8 Performance analysis of graph-based technique for TAS problem in terms of sum rate in 61-node network.

single communication node in each cell was adopted. It is worth noting that for the case of $N=61$, the centralized technique was replaced with the alternating-coordinate technique. In this case, the set of parameters of a certain neighborhood are coordinated while holding others fixed. The precoding matrix codebooks defined in (11) and (12) were used as the parameter set for TAS and beam selection, respectively. As for the MIMO setup, each transmitter has $N_{t}=3$ available transmit antennas and $N_{s}=2$ data streams to be transmitted, and each receiver has $N_{r}=2$ receive antennas. To obtain more realistic results, each MIMO channel response was drawn from a set containing 324,000 $2 \times 3$ channel matrices, according to (18). The parameter initialization is at random, i.e., nodes pick one of the PMIs randomly at the beginning of each simulation run in the greedy technique. In the graph-based approach, the initial messages defined in (6) are equal to zero. The maximum number of iterations $\lambda$ in each simulation run is 100 . Finally, a total of 1,000 runs were conducted for statistical purposes for the seven-node network case. Due to the fact that the number of channel data samples available is limited, a total of only 85 runs were conducted for the 61-node network case. It is worth mentioning that the channel responses were kept constant during the $\lambda$ iterations. These simulation parameters above were adopted for both simultaneous and random message-passing schedulers. As for random scheduling, the parameter $P_{\text {active }}$ was set to $90 \%, 70 \%$, or $30 \%$. Table 2 lists the simulation parameters.

\section{Example 1: transmit antenna selection}

For the TAS example, the number of radio frequency chains is 2. Consequently, the parameters to be coordinated are three PMIs for all the cells. Such parameters index the elements of the codebook in (11). Next, simulation results considering a network with seven cells, each one with a communication node, are presented.

\section{TAS and $N=7$ nodes}

The graph-based technique appears not to be affected by the different types of message-passing scheduling in terms of sum rate. Figure 6 shows that the proposed method

Table 5 Percent gain in sum rate for the TAS problem in a 61-node network

\begin{tabular}{lcccc}
\hline \% Gain in sum rate over greedy & & \multicolumn{3}{c}{ Message-passing scheduling } \\
\cline { 3 - 4 } & Simultaneous & $\mathbf{9 0 \%}$ Random & $\mathbf{6 0 \% ~ R a n d o m ~}$ & 30\% Random \\
\hline 50th CDF percentile & 1.459 & 1.459 & 1.459 \\
\hline
\end{tabular}




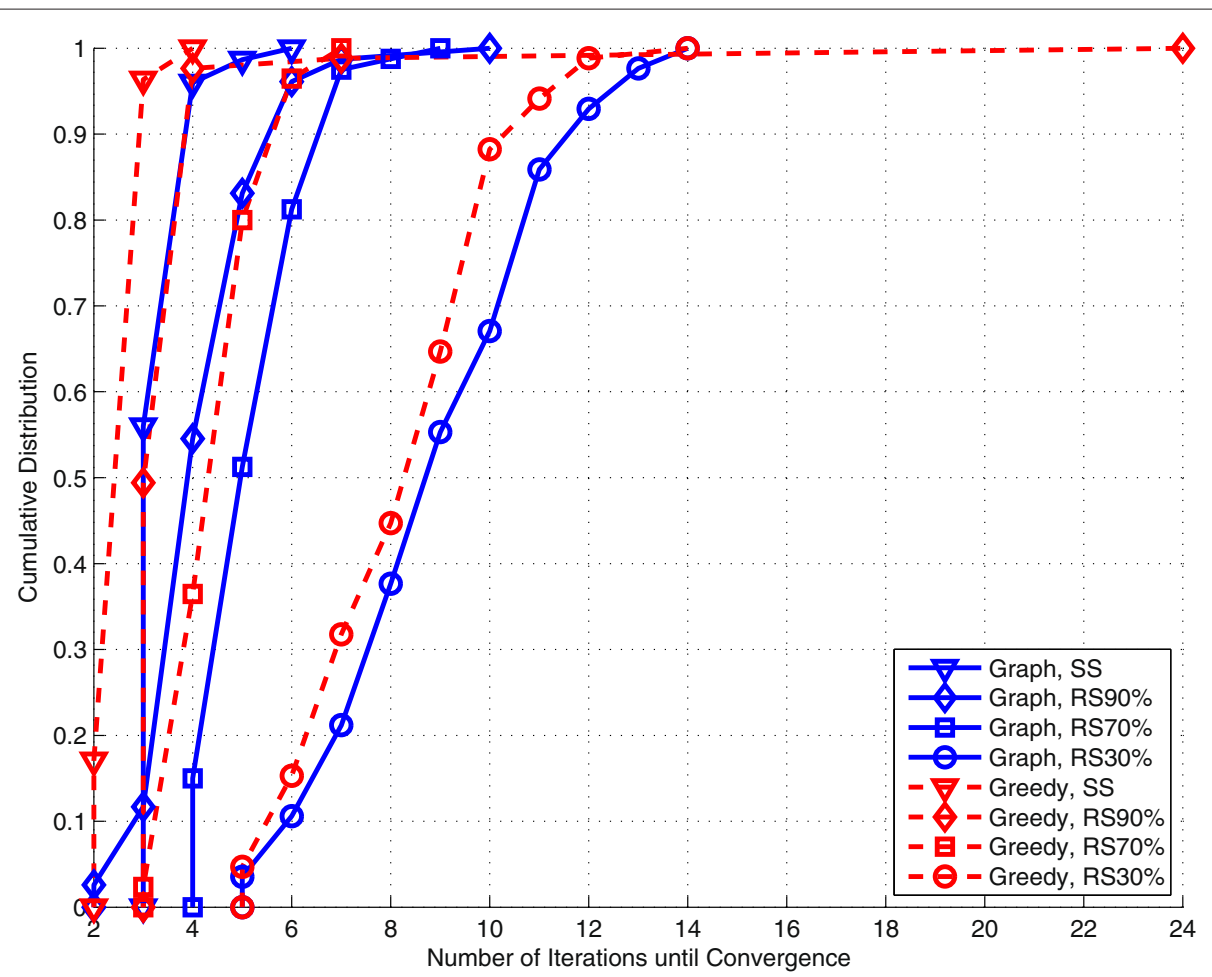

Figure 9 Convergence speed of graph-based technique against greedy technique for TAS problem in 61-node network.

in all the scheduling modes provides approximately the same performance. The maximum achievable sum rate is about 49 bits per channel use, reached by the centralized approach and also by the graph-based technique. From the curves in Figure 6, the graph-based technique approaches the optimal solution in all the simulation runs, reaching the global optimum in most of them. Clearly, the graph-based technique outperforms the greedy one.

The greedy technique is also not significantly affected by the different types of message-passing scheduling in terms of sum rate. According to Figure 6, the greedy technique with any of the scheduling modes reaches the sum rate value of 48 bits per channel use or less with $100 \%$ probability, while both the proposed and the centralized technique provide about 49 bits per channel use.

According to the results, the gain in sum rate obtained by the graph-based technique over the greedy one is largely independent of the type of scheduling. For instance, from the 50th CDF percentile, such a gain is about $2.4 \%$ for all the message-passing scheduling modes.
Table 3 shows the statistics above. Despite the small gain observed over the greedy, the proposed method provides a solution near (or equal to) the optima.

It is clear that the TAS with both greedy and graphbased solutions outperforms the random choice approach. However, the maximum gain obtained over the random choice is around $9 \%$, which may be considered too small for a precoder selection technique.

With respect to convergence speed, the proposed method with random scheduling converges faster than that with simultaneous scheduling. Figure 7 shows that the higher the probability of being active, the higher the speed of convergence. The same behavior occurs for the greedy technique. However, it converges faster than the graph-based. Both greedy and graph-based techniques converged in ten iterations or less with 100\% probability.

In terms of convergence rate, the proposed method has a different rate for each message-passing scheduling mode. For instance, the graph-based technique with simultaneous scheduling converges with $98 \%$ probability. Further,

Table 6 Convergence rate for the TAS problem in a 61-node network

\begin{tabular}{lcccc}
\hline \multirow{2}{*}{ Technique } & SS & \multicolumn{3}{c}{ Message-passing scheduling } \\
\cline { 2 - 5 } & 0.882 & RS, $\boldsymbol{P}_{\text {active }}=\mathbf{0 . 9}$ & RS, $\boldsymbol{P}_{\text {active }}=\mathbf{0 . 7}$ & RS, $\boldsymbol{P}_{\text {active }}=\mathbf{0 . 3}$ \\
\hline Graph-based & 0.905 & 0.941 & 1 \\
Greedy & 0.964 & 1 & 1 & 1 \\
\hline
\end{tabular}




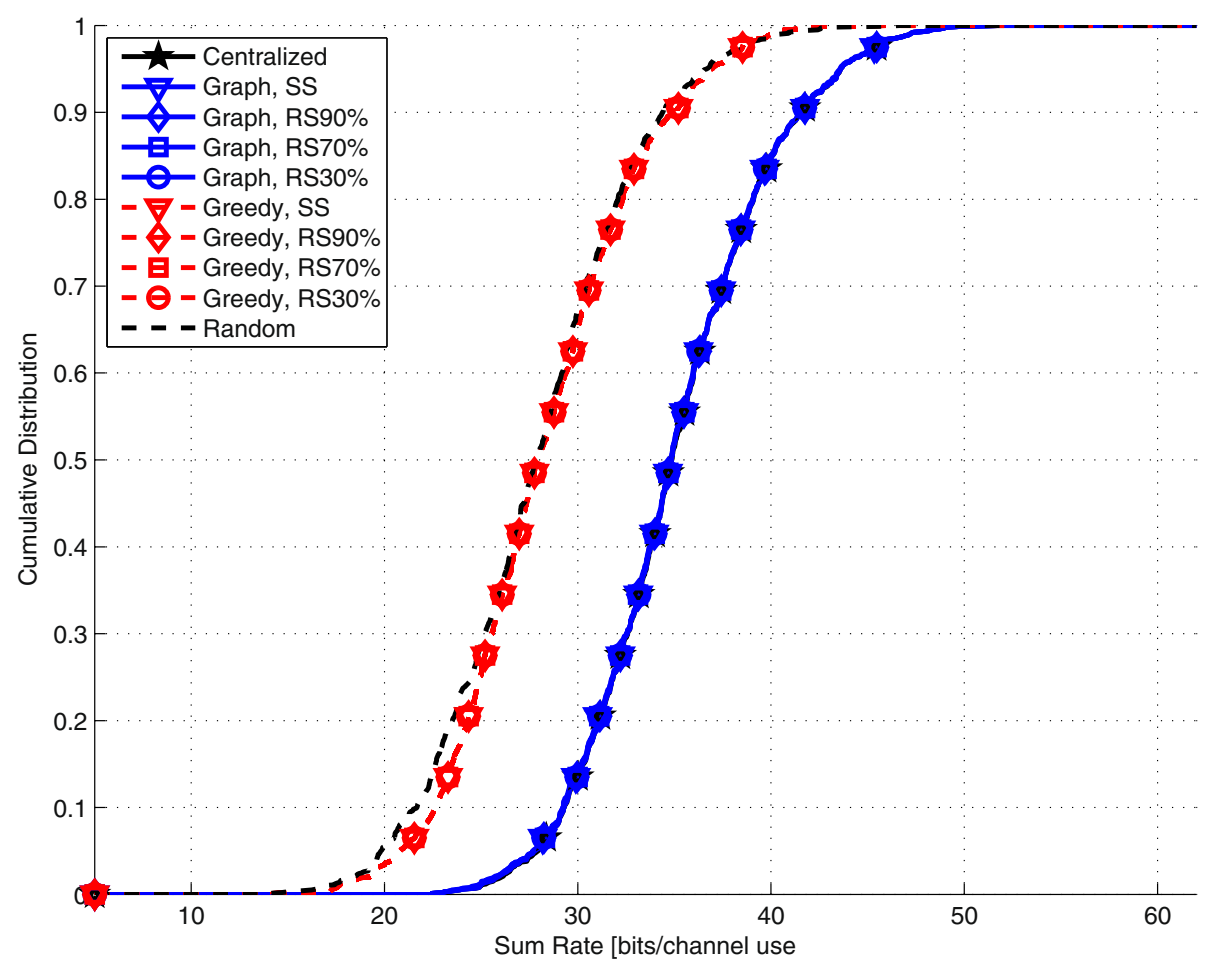

Figure 10 Performance analysis of graph-based technique for beam selection problem in terms of sum rate in seven-node network.

the proposed method with random scheduling converges with $98.5 \%$ when $P_{\text {active }}=0.9,99.3 \%$ probability when $P_{\text {active }}=0.7$, and $100 \%$ probability when $P_{\text {active }}=0.3$. Finally, the greedy technique with random scheduling converges with $100 \%$ probability, while it converges with 99.6\% probability in the simultaneous scheduling mode. Table 4 shows all the statistics regarding convergence rate. Interestingly, even when the proposed method does not converge, it provides a near-optimal solution, as expected. In the following, simulation results considering a larger network with $N=61$ are presented.

\section{TAS and $N=61$ nodes}

Increasing the network size to 61 nodes, the graph-based technique is still not affected by the different modes of message-passing scheduling in terms of sum rate. Accordingly, Figure 8 shows that all the scheduling modes make the proposed method to have approximately the same performance. For this network size, the centralized approach is properly replaced with the alternating-coordinate technique, which provides the 'best' solution in this case.
It reaches a maximum sum rate of about 231 bits per channel use, followed by the graph-based technique. In Figure 8, the proposed method approaches the best solution in all the simulation runs (often reaching the best) and outperforms the greedy technique. Moreover, the proposed method appears to be scalable since it maintains its good performance in larger networks. The greedy technique is also not significantly affected by the different types of message-passing scheduling, but as shown in Figure 8, the maximum achievable sum rate for such an approach is around 227 bits per channel use.

In general, the gain in sum rate obtained by the graphbased technique over the greedy one is largely independent of the scheduling mode in terms of sum rate. For instance, from the 50th CDF percentile, the gain is about $1.459 \%$ for all the scheduling modes. Again, the gain obtained over the random choice is too small, around $8 \%$. Table 5 shows the statistics above.

As for convergence speed, the graph-based technique with simultaneous scheduling converges faster than that with random scheduling, as in the previous case. Figure 9

Table 7 Percent gain in sum rate for the beam selection problem in a seven-node network

\begin{tabular}{|c|c|c|c|c|}
\hline \multirow{2}{*}{$\%$ Gain in sum rate over greedy } & \multirow[b]{2}{*}{ Simultaneous } & \multicolumn{3}{|c|}{ Message-passing scheduling } \\
\hline & & 90\% Random & $60 \%$ Random & $30 \%$ Random \\
\hline 50th CDF percentile & 24.683 & 24.755 & 24.754 & 24.754 \\
\hline
\end{tabular}




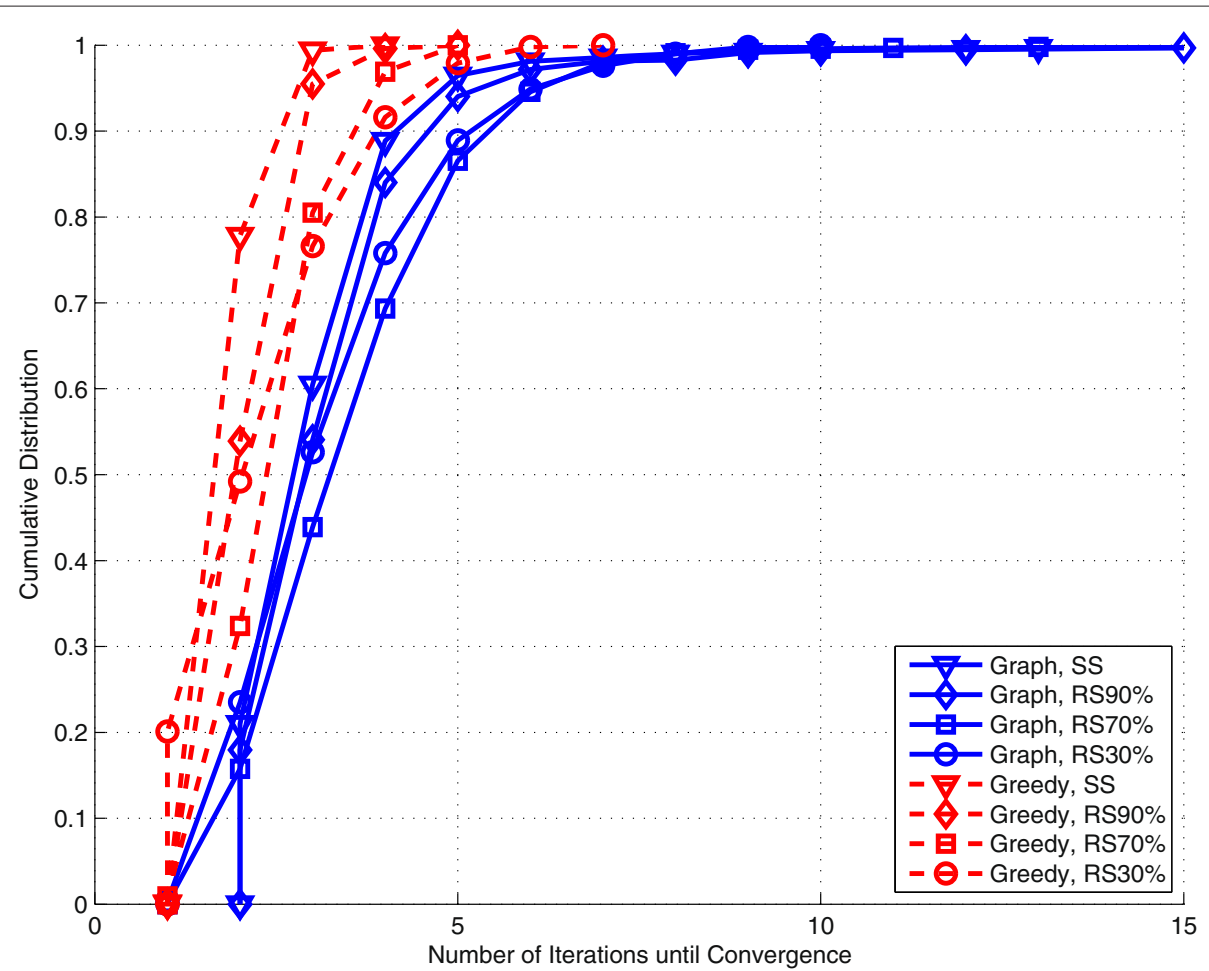

Figure 11 Convergence speed of graph-based technique against greedy technique for beam selection problem in seven-node network.

also shows that the higher the probability of node activation, the higher the speed of convergence. Again, the greedy technique has the same behavior, but it converges faster than the graph-based technique. In Figure 9, both techniques converge in up to 24 iterations.

With regard to convergence rate, the graph-based technique observes different rates, which depends on the message-passing scheduling mode. For instance, such an approach with simultaneous scheduling converges with $88.2 \%$ probability. However, considering random scheduling, it converges with $90.5 \%$ probability when $P_{\text {active }}=0.9$, with $94.1 \%$ probability when $P_{\text {active }}=0.7$, and with $100 \%$ when $P_{\text {active }}=0.3$. Differently, the greedy technique converges with $100 \%$ probability in all the scheduling modes except in the simultaneous scheduling mode, where it converges with $96 \%$ probability. Table 6 shows all the statistics above regarding convergence rate.

\section{Example 2: fixed-beam selection}

For the beam selection problem, the parameters to be coordinated are also three PMIs for all the cells. Such parameters index the elements of the codebook in (12). Next, simulation results considering a network with seven cells, each one with a communication node, are presented.

\section{Beam selection and $N=7$ nodes}

Similar to the previous example, the graph-based technique appears not to be affected by the different modes of message-passing scheduling in terms of sum rate. In Figure 10, the proposed method has approximately the same performance in all the scheduling modes. The maximum achievable sum rate is about 51 bits per channel use, reached by both the centralized and graph-based techniques. From the curves in Figure 10, the graph-based technique approaches the optimal solution in all the simulation runs, reaching the global optimum in most of them. Once more, the proposed method provides a near-optimal solution. As expected, the graph-based technique outperforms the greedy and also the random choice with a large gain in sum rate.

The greedy technique is not affected by the different types of message-passing scheduling in terms of sum rate.

Table 8 Convergence rate for the beam selection problem in a seven-node network

\begin{tabular}{|c|c|c|c|c|}
\hline \multirow{2}{*}{ Technique } & \multirow[b]{2}{*}{ Simultaneous } & \multicolumn{3}{|c|}{ Message-passing scheduling } \\
\hline & & $90 \%$ Random & $60 \%$ Random & $30 \%$ Random \\
\hline Graph-based & 0.906 & 0.919 & 0.959 & 1 \\
\hline Greedy & 1 & 1 & 1 & 1 \\
\hline
\end{tabular}




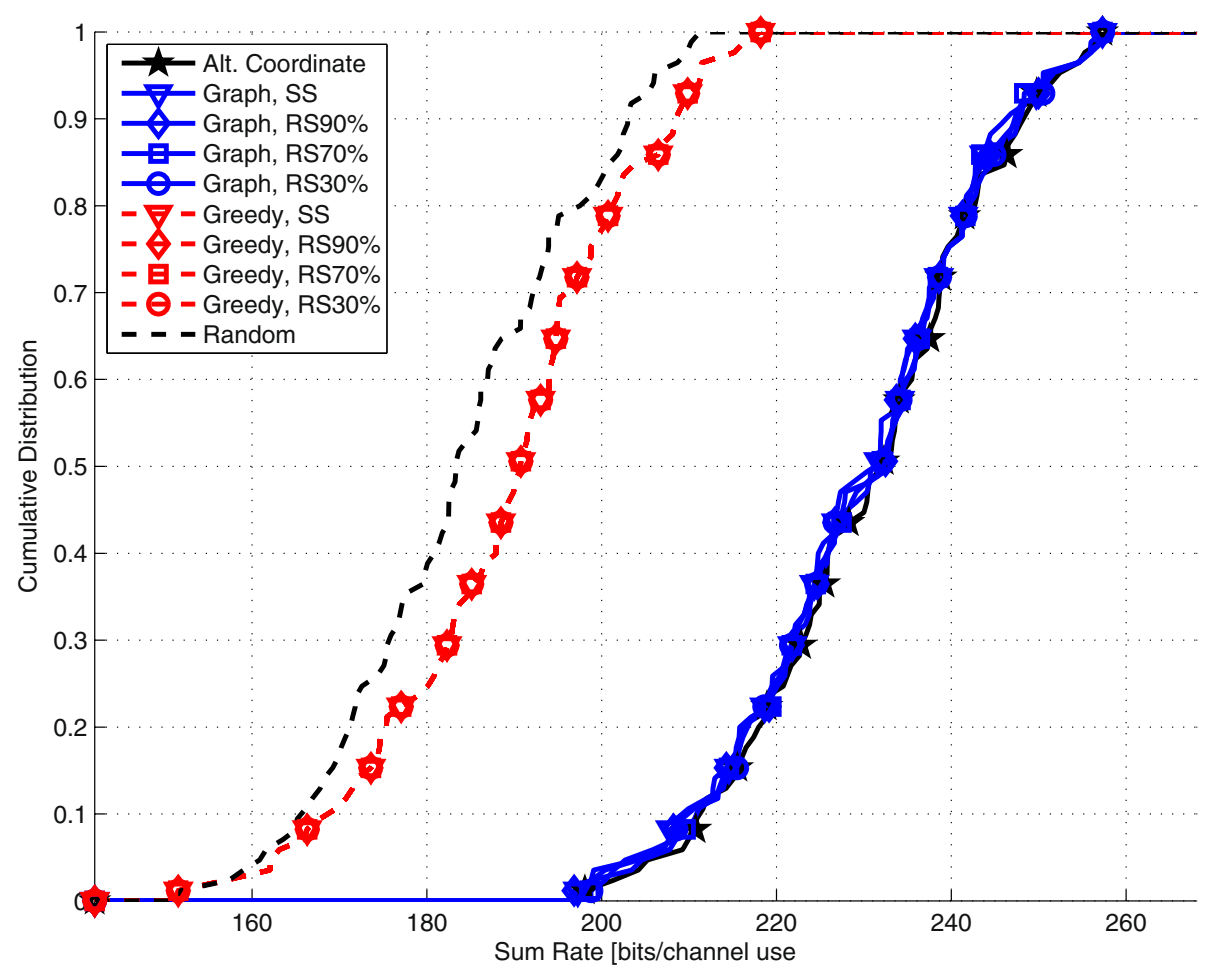

Figure 12 Performance analysis of graph-based technique for beam selection problem in terms of sum rate in 61-node network.

Still in Figure 10, the greedy technique with any of the scheduling modes reaches the sum rate value of 45 bits per channel use or less with $100 \%$ probability, while both the proposed and the centralized technique provide about 51 bits per channel use as aforementioned. Figure 10 also shows that the greedy technique poorly outperforms the random choice.

Based on the curves in Figure 10, the gain in sum rate obtained by the graph-based technique over the greedy is largely independent of the mode of scheduling. For instance, from the 50th CDF percentile, such a gain is around $24.7 \%$ for all the message-passing scheduling modes. Table 7 shows the statistics above. Dissimilarly to the previous example, the gain observed over the greedy and the random choice is significantly large.

As for convergence speed, the proposed method technique with random scheduling converges faster than that with simultaneous scheduling. Figure 11 shows that the higher the probability of being active, the higher the speed of convergence. Again, the greedy technique behaves the same way, i.e., converges faster than the graph-based technique. Both the greedy and graph-based techniques converge in 15 iterations or less with $100 \%$ probability.

In terms of convergence rate, the graph-based technique has a different rate for each message-passing scheduling mode adopted. For instance, the graph-based technique with simultaneous scheduling converges with $90.6 \%$ probability. Further, the proposed method with random scheduling converges with $91.9 \%$ when $P_{\text {active }}=0.9$, $95.9 \%$ probability when $P_{\text {active }}=0.7$, and $100 \%$ probability when $P_{\text {active }}=0.3$. On the other hand, the greedy technique converges with $100 \%$ probability with any of the message-passing scheduling types. Table 8 shows all the statistics regarding convergence rate. Once again, even when the proposed method does not converge, it provides a near-optimal solution, as expected.

\section{Beam selection and $N=61$ nodes}

Considering a network size of 61 nodes, the graph-based technique is still not affected by the different modes of message-passing scheduling in terms of sum rate. Such a behavior is shown in Figure 12, where all the scheduling

Table 9 Percent gain in sum rate for the beam selection problem in a 61-node network

\begin{tabular}{|c|c|c|c|c|}
\hline \multirow{2}{*}{$\%$ Gain in sum rate over greedy } & \multirow[b]{2}{*}{ Simultaneous } & \multicolumn{3}{|c|}{ Message-passing scheduling } \\
\hline & & 90\% Random & $60 \%$ Random & $30 \%$ Random \\
\hline 50th CDF percentile & 21.449 & 21.868 & 21.644 & 21.707 \\
\hline
\end{tabular}




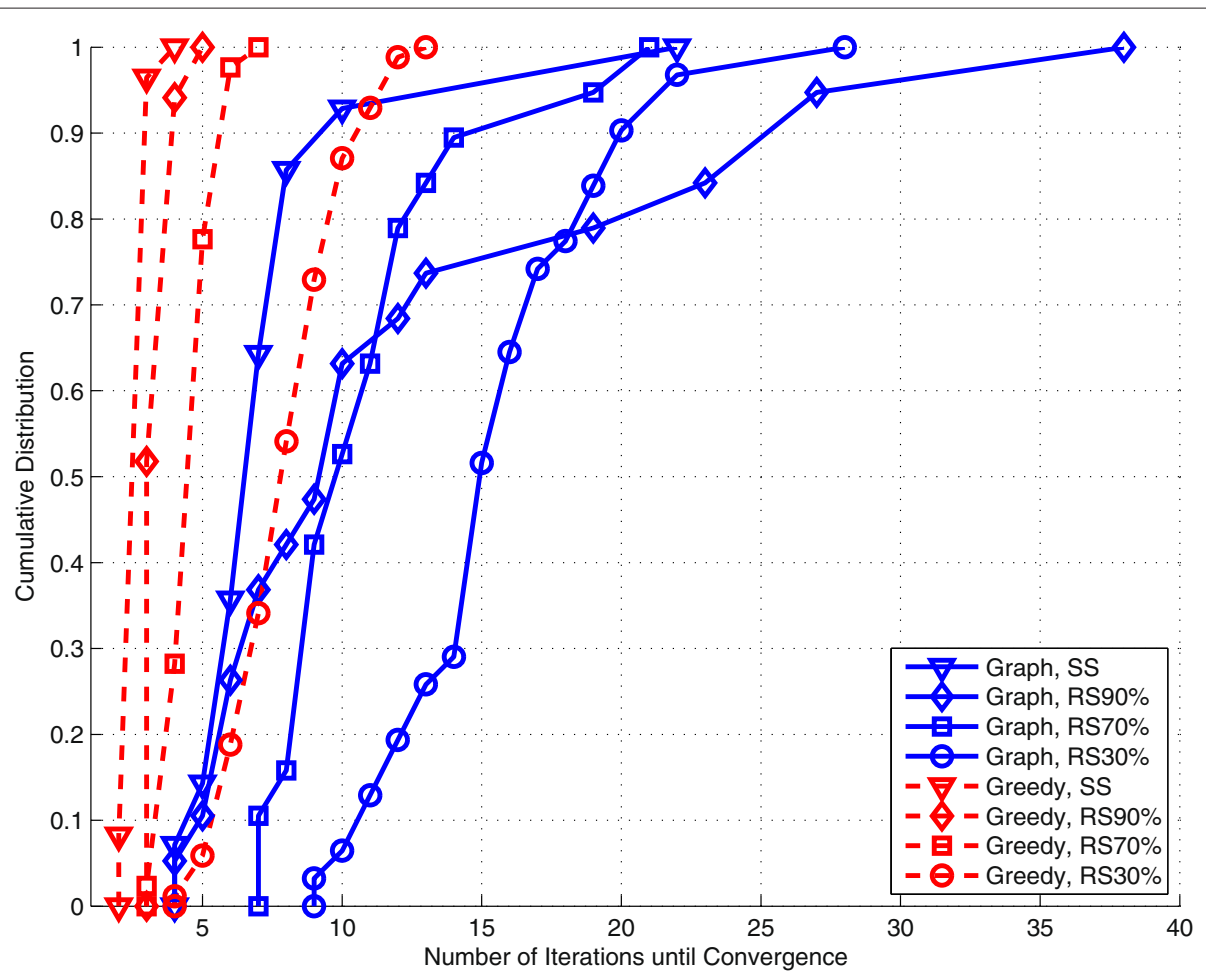

Figure 13 Convergence speed of graph-based technique against greedy technique for beam selection problem in 61-node network.

modes make the proposed method to have approximately the same performance. Properly, the centralized approach is replaced with the alternating-coordinate technique, which reaches a maximum sum rate of about 257 bits per channel use, followed by the graph-based technique. In Figure 12, the proposed method approaches the solution of the alternating-coordinate technique in all the simulation runs (often reaching that result) and outperforms the greedy technique. Thus, the proposed method maintains its good performance even in such a larger network. The greedy technique is not significantly affected by the different modes of message-passing scheduling, but Figure 12 shows that it achieves a lower maximum sum rate of 218 bits per channel use. Again, the random choice is greatly outperformed by the proposed technique.

As observed in all the examples above, the gain in sum rate obtained by the proposed method over the greedy technique is independent of scheduling mode in terms of sum rate. For example, from the 50th CDF percentile, the gain is around $21 \%$ for all the scheduling modes. Table 9 shows the statistics above.
In terms of convergence speed, the graph-based technique with the simultaneous scheduling converges faster than that with random scheduling, as in all the previous cases. For both the graph-based and greedy techniques, Figure 13 shows that the higher the probability of node activation, the higher the speed of convergence. In Figure 13, both techniques converge in up to 38 iterations. Nevertheless, the greedy technique converges faster than the graph-based.

As for convergence rate, the graph-based technique observes different rates, which depends on the messagepassing scheduling mode. However, in this example, the proposed method shows small convergence rates. For instance, the graph-based approach with simultaneous scheduling converges with $16.4 \%$ probability. Considering random scheduling, it converges with $22.3 \%$ probability when $P_{\text {active }}=0.9$, with $22.3 \%$ probability when $P_{\text {active }}=0.7$, and with $36.4 \%$ when $P_{\text {active }}=0.3$. On the contrary, the greedy technique converges with $100 \%$ probability in all the scheduling modes. Table 10 shows all the statistics above regarding convergence rate. The

Table 10 Convergence rate for the beam selection problem in a 61-node network

\begin{tabular}{lcccc}
\hline \multirow{2}{*}{ Technique } & Simultaneous & \multicolumn{3}{c}{ Message-passing scheduling } \\
\cline { 3 - 5 } & 0.164 & $\mathbf{9 0 \% \text { Random }}$ & $\mathbf{6 0 \% \text { Random }}$ & $\mathbf{3 0 \% \text { Random }}$ \\
\hline Graph-based & 1 & 0.223 & 0.223 & 0.364 \\
Greedy & 1 & 1 & 1 \\
\hline
\end{tabular}


small number of simulation runs in this example may have led to those small rates, but despite those rates, the proposed method has a similar performance compared to the alternating-coordinate technique and also outperforms the greedy solution with large gain in sum rate.

\section{Summary of the results}

The small observed gain in the TAS problem is likely due to the choice of the particular precoder codebook of antenna selection. The selection of transmit antennas at a certain node seems not to significantly affect the selection made by the others and, consequently, poorly mitigates mutual interference observed by nodes. Therefore, even the simpler greedy solution may achieve a good result. Besides, the comparison of the TAS with the random choice shows that it does not have a great impact on the performance of the nodes since it provides gains in sum rate below $10 \%$.

In the fixed-beam selection problem, the large observed gain is likely due to the use of directional beamforming precoding matrices. The selection of fixed beams at a certain node seems to impact on the selection made by the others since it may directly avoid a node causing interference to its neighbors. Thus, the graph-based technique can obtain a better solution than the greedy one based on the fact that the messages exchanged among nodes regard the impact of nodes' decisions on their neighboring nodes.

In general, the proposed method reaches the global optima efficiently, which means lower computational complexity and less backhaul traffic, compared with the centralized solution, and always outperforms the greedy solution and, consequently, the random choice approach. Additionally, the use of different modes of message-passing scheduling does not impact on the solution in terms of sum rate but affects convergence speed and convergence rate. Simultaneous scheduling leads to faster-but-less-often convergence as all the nodes exchange messages. Random scheduling makes the algorithms to converge more frequently and demand more iterations to converge, but less nodes participate in the message-passing procedure. Therefore, the choice of the message-passing scheduling might depend on the network constraints, such as low latency and limited backhaul traffic.

\section{Conclusions}

The graph-based method for distributed parameter coordination considers the impact of nodes' decisions on their neighboring nodes. The information (message) exchange is only among neighbors. Such a technique reaches the (near) optimal solution more frequently than the greedy approach at cost of larger message size and slower convergence. The use of random scheduling in the problems of precoder selection tends to enhance the convergence rate but decreases the convergence speed. It is worthwhile to note that the graph-based approach is totally adaptable to any discrete problem of parameter coordination and any network size. As for the numerical results, the graphbased technique provides good gains in the global cost over the greedy solution, specially in the beam selection problem. As future studies, one may think of working on message-passing scheduling with faster convergence and message exchange with reduced message size.

\section{Competing interests}

The authors declare that they have no competing interests.

\section{Acknowledgments}

This work was partially supported by the Innovation Center, Ericsson Telecomunicações S.A., Brazil, and National Council for Scientific and Technological Development (CNPq). The authors would like to thank Jiann-Ching Guey for valuable discussions and Ericsson Research for providing the measurement data.

\section{Author details}

${ }^{1}$ Wireless Telecommunication Research Group (GTEL), Universidade Federal do Ceará (UFC), Fortaleza 60455-760, Brazil. ${ }^{2}$ Ericsson Research, San José, CA 95134, USA.

Received: 15 June 2012 Accepted: 28 March 2013

Published: 19 April 2013

\section{References}

1. Z Han, K Liu, Resource Allocation for Wireless Networks: Basics, Techniques, and Applications. (Cambridge University Press, New York, 2008)

2. A Paulraj, R Nabar, D Gore, Introduction to Space-Time Wireless Communications. (Cambridge University Press, Cambridge, 2003)

3. TRappaport, Wireless Communications: Principles and Practice, 2 nd edn (Prentice Hall, Upper Saddle River, 2001)

4. J Andrews, W Choi, R Heath, Overcoming interference in spatial multiplexing MIMO cellular networks. IEEE Wireless Commun. 14(6), 95-104 (2007)

5. F Kschischang, B Frey, HA Loeliger, Factor graphs and the sum-product algorithm. IEEE Trans. Inf. Theory. 47(2), 498-519 (2001)

6. T Başar, GJ Olsder, Dynamic, Noncooperative Game Theory. (Harcourt Brace \& Company, London, 1995)

7. ISohn, SH Lee, J Andrews. A graphical model approach to downlink cooperative MIMO systems. In 2010 IEEE Global Telecommunications Conference (GLOBECOM 2010). Miami, 6-10 Dec 2010, pp. 1-5

8. I Sohn, SH Lee, J Andrews, Belief propagation for distributed downlink beamforming in cooperative MIMO cellular networks. IEEE Trans. Wireless Commun. 10(12), 4140-4149 (2011)

9. BL Ng, J Evans, S Hanly, D Aktas. Transmit beamforming with cooperative base stations. In 2005 IEEE International Symposium on Information Theory (ISIT 2005). Adelaide, 4-9 Sept 2005,pp. 1431-1435

10. BL Ng, J Evans, S Hanly, D Aktas, Distributed downlink beamforming with cooperative base stations. IEEE Trans. Inf. Theory. 54(12), 5491-5499 (2008)

11. JRW Heath, D Love, Multimode antenna selection for spatial multiplexing systems with linear receivers. IEEE Trans. Signal Process. 53(8), 3042-3056 (2005)

12. S Aji, R McEliece, The generalized distributive law. IEEE Trans. Inf. Theory. 46(2), 325-343 (2000)

13. I Guerreiro, C Cavalcante. A distributed approach for antenna subset selection in MIMO systems. In 20107 th International Symposium on Wireless Communication Systems (ISWCS 2010). York, 19-22 Sept 2010, pp. 199-203

14. J Lee, JK Han, J Zhang, MIMO technologies in 3GPP LTE and LTE-advanced. EURASIP J. Wireless Commun. Netw. 2009, 3:1-3:10 (2009)

15. F Kschischang, B Frey, Iterative decoding of compound codes by probability propagation in graphical models. IEEE J. Selected Areas Commun. 16(2), 219-230 (1998)

16. E Telatar, Capacity of multi-antenna Gaussian channels. Eur. Tran. Telecommun. 10, 585-595 (1999) 
17. G Scutari, DP Palomar, S Barbarossa, Optimal linear precoding strategies for wideband noncooperative systems based on game theory - part I: Nash equilibria. IEEE Trans. Signal Process. 56(3), 1230-1249 (2008)

18. Y Selen, H Asplund. $3 G$ LTE simulations using measured MIMO channels. In IEEE Global Telecommunications Conference, 2008 (IEEE GLOBECOM 2008), New Orleans, 30 Nov-4 Dec 2008, pp. 1-5

19. M Binelo, ALF De Almeida, J Medbo, H Asplund, FRP Cavalcanti. MIMO channel characterization and capacity evaluation in an outdoor environment. In 2010 IEEE 72nd Vehicular Technology Conference Fall (VTC 2010-Fall). Ottawa, 6-9 Sept 2010, pp. 1-5

20. M Ozcelik, N Czink, E Bonek. What makes a good MIMO channel model? In 2005 IEEE 61st Vehicular Technology Conference, 2005 (VTC 2005-Spring), vol. 1, Stockholm, 30 May-1 June 2005, pp. 156-160

doi:10.1186/1687-6180-2013-83

Cite this article as: Guerreiro et al: A distributed approach to precoder selection using factor graphs for wireless communication networks. EURASIP Journal on Advances in Signal Processing 2013 2013:83.

\section{Submit your manuscript to a SpringerOpen ${ }^{\mathcal{O}}$ journal and benefit from:}

- Convenient online submission

- Rigorous peer review

- Immediate publication on acceptance

- Open access: articles freely available online

- High visibility within the field

- Retaining the copyright to your article

Submit your next manuscript at $\gg$ springeropen.com 